$$
\text { "kovacs" — 2008/4/9 — 10:31 — page } 407 \text { — \#1 }
$$

\title{
Blind versus wise use of CAS
}

\author{
ZOLTÁN KOVÁCS
}

Abstract. During my courses for mathematics major students I often use technology linked to the arising problems. In such cases I noted that some students were used to learn just some procedures, which made them able to solve (partially) some problems and when they got the result, they accepted it passively and did not relate it to the initial problem.

In this paper I outline a strategy and investigate some simple exercises about how to develop a critical attitude towards the results obtained by technology in an introductory course to CAS.

I believe that wise use of technology offers an effective method in teaching mathematics, without reducing the students' mental contribution.

Key words and phrases: computer algebra systems in learning mathematics.

ZDM Subject Classification: R25, R35.

\section{Introduction}

Approximately for a decade I deliver courses for mathematics major students (specialized in applied mathematics) on using computer algebra systems to solve mathematical problems. One of them is an introductory course and its syllabus covers an overall introduction to CAS and problem solving in linear algebra. Another course is Computer Geometry. Moreover I often use technology related to the arising problems in other courses. (Now we have Maple 9 in the lab and every year I spend some time on Maxima, too.) The purely computational aspects of CAS do not arise any problems in my experience, in contrast with the problems that require deeper mathematical understanding. During my courses I

Copyright (C) 2007 by University of Debrecen 


$$
\text { "kovacs" — 2008/4/9 — 10:31 — page } 408 \text { — \#2 }
$$

have noticed that some students were used to learn just some procedures, which made them able to solve (partially) some problems and when they got the result, they accepted it passively and did not relate it to the initial problem.

In this paper I outline a strategy about how to develop a more critical attitude towards the results obtained from technology in an introductory course, at a very early stage of using CAS.

I believe that wise use of technology offers a modern method in teaching mathematics, without reducing the students' mental contribution.

\section{2. 'Be paranoid' or believing what you see?}

The section title is quoted from [1, p. 43].

Using a CAS we trust in the correct implementation of mathematical theory integrated in the system. However, we all make mistakes and even well known mathematicians make mistakes as well. To err is human... Some mistakes appear even in the computer algebra systems.

Here is an example of what can go wrong when one differentiates under the integral sign without justification. In 1815 Cauchy obtained the convergent integral

$$
\int_{x=0}^{\infty} \sin \left(x^{2}\right) \cdot \cos (s x) d x=\frac{1}{2} \sqrt{\frac{\pi}{2}}\left[\cos \left(\frac{s^{2}}{4}\right)-\sin \left(\frac{s^{2}}{4}\right)\right]
$$

He then differentiated under the integral sign with respect to $s$ and obtained

$$
\int_{x=0}^{\infty} x \sin \left(x^{2}\right) \cdot \sin (s x) d x^{6}=, \frac{s}{4} \sqrt{\frac{\pi}{2}}\left[\sin \left(\frac{s^{2}}{4}\right)+\cos \left(\frac{s^{2}}{4}\right)\right] .
$$

This divergent integral have been reproduced ever since and in [5] it was reported that Maple (V.5.) and Mathematica (4.0) had difficulties with these integrals. (Mathematica thought (2) converge.) Now, Maple gives the correct answer. Note that Maxima 0.7.1 cannot handle (2) and gives it back unevaluated.

The only strategy to handle these problems is checking. 'Check your results in at least two ways (the more the better). ... do a simple case by hand, do selected cases numerically, substitute your solution back into the defining equation...' $([1$, p. 43$])$.

There are many possibilities for applying this strategy for example in linear algebra. For beginners I always require both the 'step by step' solution and 'one command solution', moreover the students always have to substitute the 


$$
\text { "kovacs" — 2008/4/9 — 10:31 — page } 409-\# 3
$$

result into the original problem. (Topics: Gauss elimination, solving linear system of equations, eigenvalue-problem, normal forms.) Here I review an instructive parametric example.

EXERCISE 1. Find parameter $t$ so that the following system of linear equations has one dimensional solution space.

$$
\begin{aligned}
x_{1}+x_{2}+t x_{3} & =2 \\
x_{1}+t x_{2}+x_{3} & =-1 \\
t x_{1}+x_{2}+x_{3} & =-1 .
\end{aligned}
$$

The base matrix of $(3)$ is

$>A:=\operatorname{matrix}([[1,1, t],[1, t, 1],[t, 1,1]])$;

$$
A:=\left[\begin{array}{lll}
1 & 1 & t \\
1 & t & 1 \\
t & 1 & 1
\end{array}\right]
$$

For rank $A$ linalg package of Maple 9 gives 3! This 'result' would imply that the system always has a unique solution. For $t=1$ the system has evidently no solution. Maple is wrong. (Note that the LinearAlgebra package leaves rank (A) unevaluated.) For $\operatorname{det}(A)$ it gives the correct answer $-t^{3}+3 t-2$. Solutions of the equation $-t^{3}+3 t-2=0$ are $(-2,1,1)$. For $t=-2$ :

$>\mathrm{B}:=\operatorname{eval}(\mathrm{A}, \mathrm{t}=-2)$;

$$
B:=\left[\begin{array}{ccc}
1 & 1 & -2 \\
1 & -2 & 1 \\
-2 & 1 & 1
\end{array}\right]
$$

and $\operatorname{rank}(B)=2$. It is easy to check that the system is solvable and the dimension of the solution space is 1 .

\section{Explain the result}

I try to develop the critical attitude even at the very early stage of technology skills.

The following exercises appear at the introduction of simplify and convert commands. 


$$
\text { "kovacs" — 2008/4/9 — 10:31 — page } 410 \text { — \#4 }
$$

Exercise 2. Provided $a b=1$, expand and simplify $(a+b)^{10}$. Explain the number 252 in the result.

The Maple code is

$>\operatorname{simplify}((\mathrm{a}+\mathrm{b}) \wedge 10,\{\mathrm{a} * \mathrm{~b}=1\}) ;$

and the result is

$$
a^{10}+10 a^{8}+\cdots+252 .
$$

Of course the student is expected to refer to the binomial theorem:

$$
\left(\begin{array}{c}
10 \\
5
\end{array}\right) a^{5} b^{5}=252(a b)^{5}=252 .
$$

ExERCISE 3. Convert the following trigonometric expressions to radicals: $\sin \left(\frac{\pi}{8}\right), \sin \left(\frac{\pi}{9}\right)$. Explain the results.

$>\operatorname{convert}(\sin (\mathrm{Pi} / 8)$, 'radical') ;

gives the following output

$$
\frac{\sqrt{2-\sqrt{2}}}{2}
$$

but

$>\operatorname{convert}(\sin (\mathrm{Pi} / 9)$, 'radical') ;

remains unevaluated. Answering the first question is an easy exercise which can be solved by 'paper and pencil'. The trigonometric identity

$$
2 \sin \left(\frac{\pi}{8}\right) \cos \left(\frac{\pi}{8}\right)=\sin \left(\frac{\pi}{4}\right)
$$

gives the following equation for $x=\sin (\pi / 8)$ :

$$
x \sqrt{1-x^{2}}=\frac{\sqrt{2}}{4},
$$

which reduces to a quadratic equation. Why does the second expression remain unevaluated? The question (which is equivalent to the Euclidean construction of the regular 9-gon) leads to the elementary Galois theory and requires a deeper mathematical understanding.

In some cases we have an idea concerning the result. Compare always the output with your expectations. The following open ended exercise is designed for developing this skill. 


$$
\text { "kovacs" — 2008/4/9 — 10:31 — page } 411 \text { — \#5 }
$$

Sometimes the misleading behaviour of the computer generated plots by CAS is obvious (e.g. graph of $\sin (1 / x)$, frac $(1 / x))$. Scaling is another relevant question which I demonstrate in the following part.

EXERCISE 4. Plot the functions $x \mapsto x^{3}$ and $x \mapsto 2^{x}$ in the interval $[0, \infty]$. Evaluate the plot.

$>\operatorname{plot}\left(\left[\mathrm{x}^{\wedge} 3,2^{\wedge} \mathrm{x}\right], \mathrm{x}=0\right.$..infinity);

gives Figure 1. I set this exercise in an exam. All of the students remarked

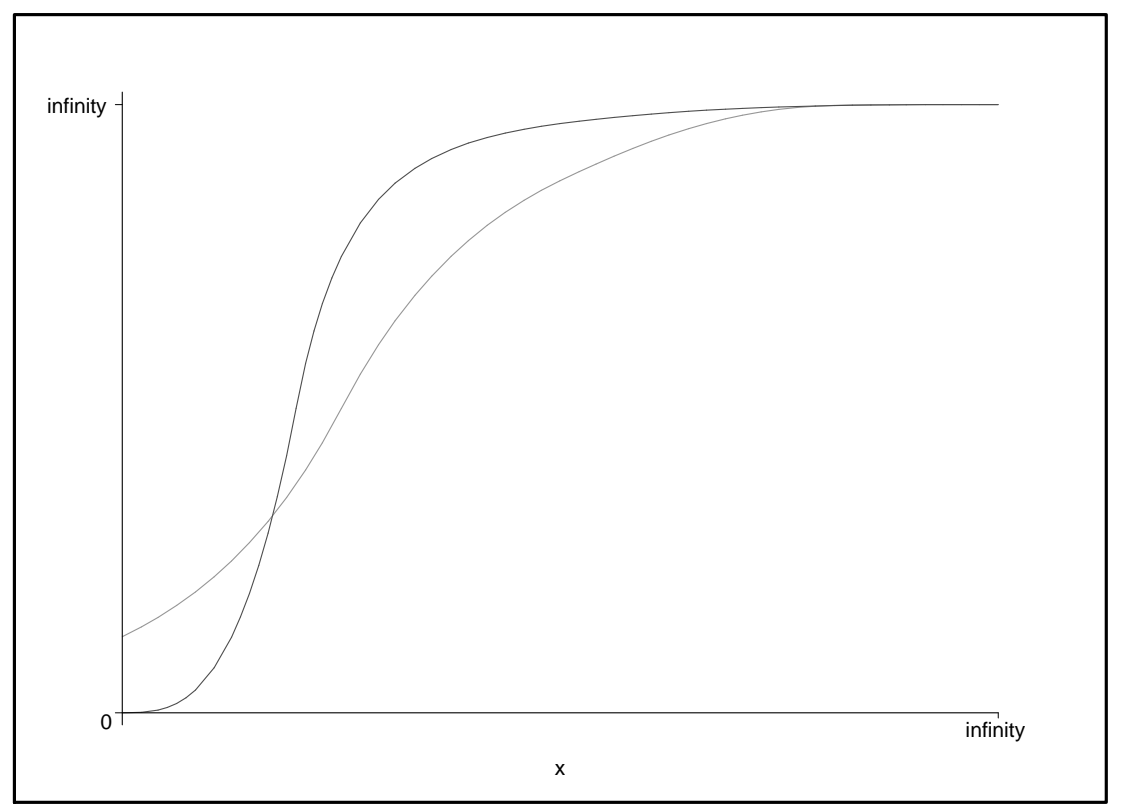

Figure 1

that the plot did not refer to the convexity of the functions. However, other aspects emerged: limit at the infinity, number of solutions of the equation $x^{3}=2^{x}$ (properly plotted); slope of the functions (the plots did not refer properly to this property). 


$$
\text { "kovacs" — 2008/4/9 — 10:31 — page } 412 \text { — \#6 }
$$

\section{CAS needs your help}

One of the most plausible topic for user-CAS interaction is 'numerical methods for finding roots of equations'. First, Maple usually does not find all the solutions, students have to determine the appropriate interval. Second, it is a great opportunity to summarize the numerical methods for finding roots and at a more advanced level students can write their own procedure.

EXERCISE 5. Find numerical values of all the solutions of this equation:

$$
\frac{x^{2}}{20}-10 x=15 \cos (x+15) .
$$

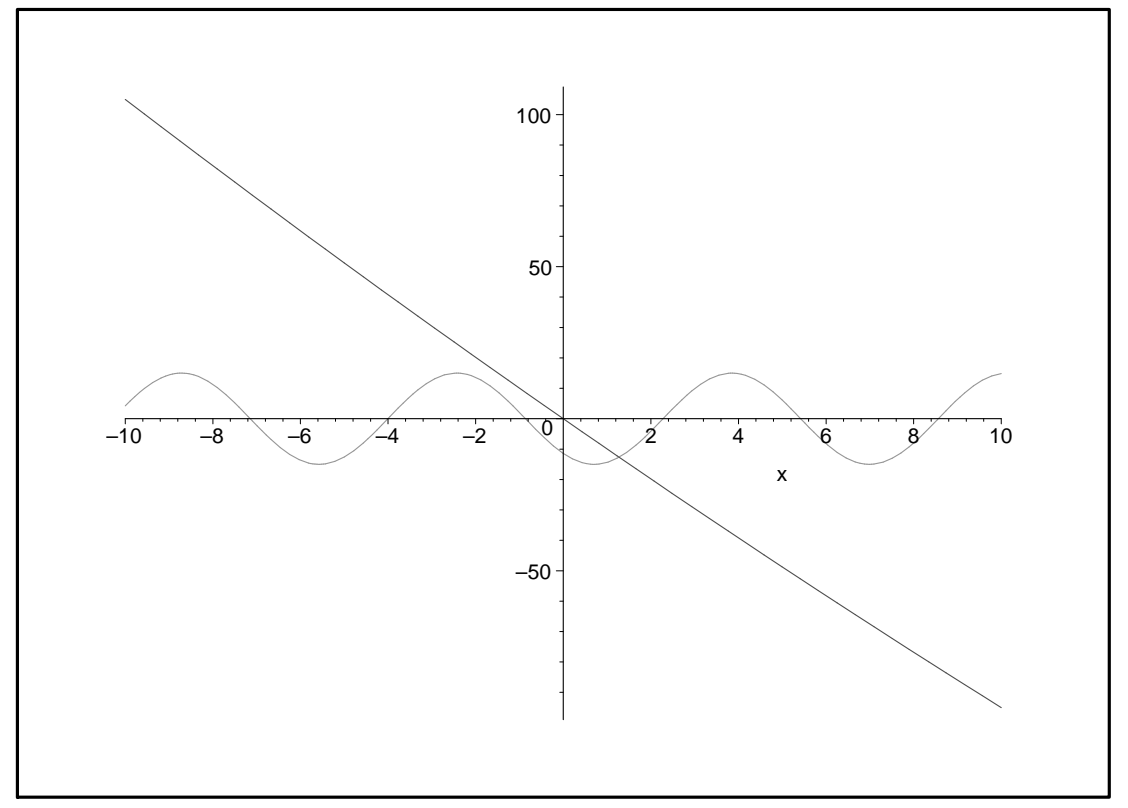

Figure 2

This is the first exercise in my course in section 'finding numerical solutions'. Maple 9 gives

$>$ fsolve $\left(x^{\wedge} 2 / 20-10 * x=15 * \cos (x+15)\right)$;

$$
1.274092075
$$

Next, the student plots a figure, in a way like this: 


$$
\text { "kovacs" — 2008/4/9 — 10:31 — page } 413 \text { — \#7 }
$$

$>\operatorname{plot}\left(\left[\mathrm{x}^{\wedge} 2 / 20-10 * x, 15 * \cos (\mathrm{x}+15)\right], \mathrm{x}=-10 \ldots 10\right)$;

(see Figure 2.) Most of my students do not recognise at first glance that at the left hand side there was a quadratic expression (see Figure 3) and around the second zero of the parabola $\left(x_{2}=200\right)$ a second root appeared:

$>$ fsolve $\left(x^{\wedge} 2 / 20-10 * x=15 * \cos (x+15), x=199 \ldots 201\right)$;

200.1193789

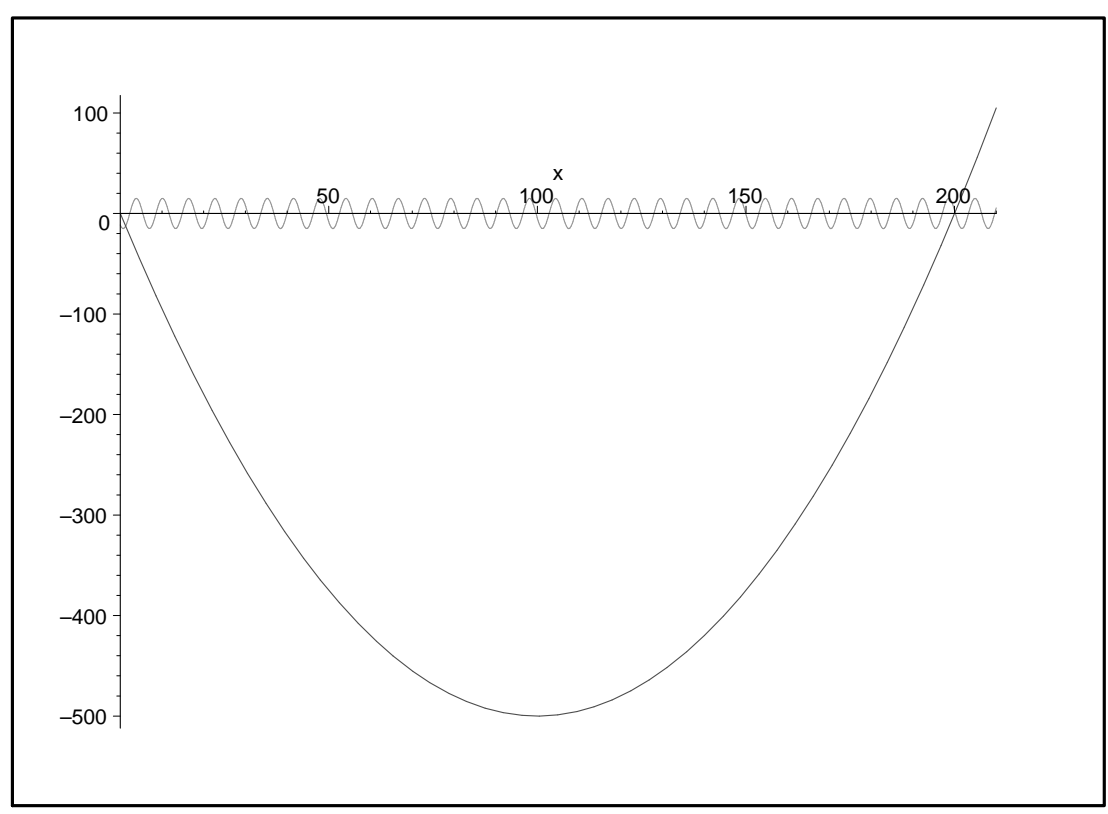

Figure 3

To test the critical attitude, in the final exam I always give a problem which is similar to the above mentioned, where the student needs some theoretical consideration to determine the appropriate interval for the numerical solution. For example:

ExERCISE 6. Find numerically the two largest solutions of the following equation:

$$
\ln (x)=5 \cdot \sin (x)
$$

In this case the best strategy is perhaps figuring out the upper limit of roots: 
$\bigoplus$

$$
\text { "kovacs" — 2008/4/9 - 10:31 — page } 414 \text { - \#8 }
$$

$>$ fsolve $(\log (x)=5, x)$;

\subsection{1}

and then finding out the interval. After some plottings (see Figure 4) one can see that $x_{\max } \in[146.1,147]$ and for the next root $x \in[145,146.1]$.

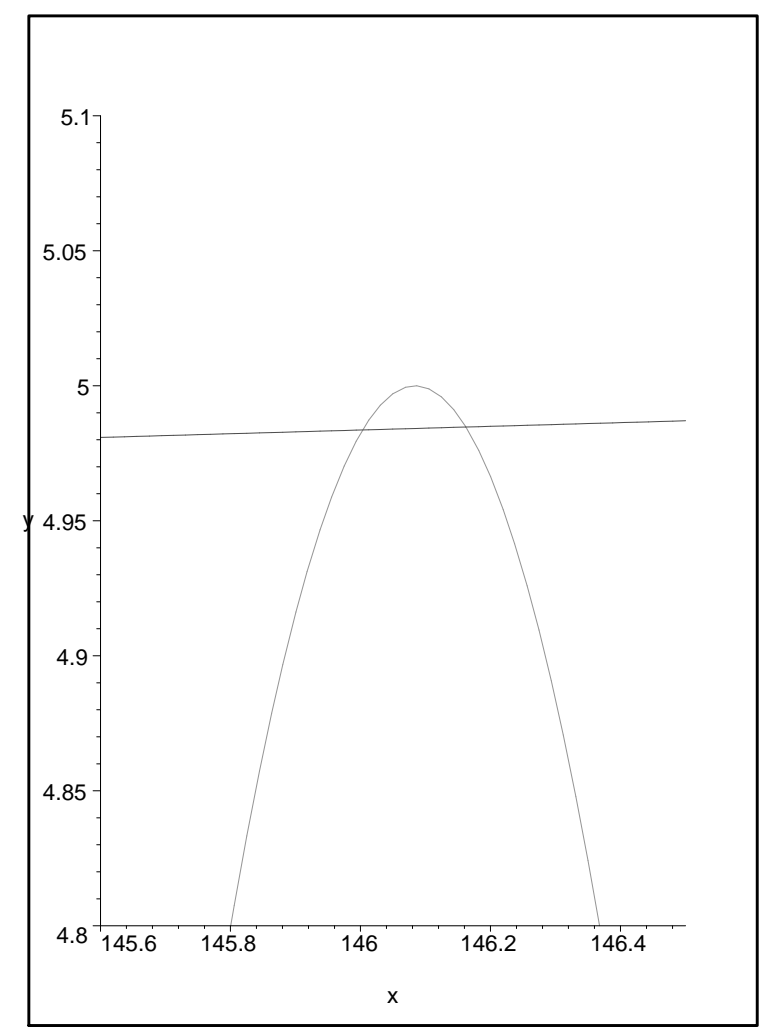

Figure 4

The following exciting example is discussed in [4].

EXERCISE 7. How many real solutions does the equation

$$
\left(\frac{1}{16}\right)^{x}=\log _{\frac{1}{16}}(x)
$$

have? 


$$
\text { "kovacs" — 2008/4/9 — 10:31 — page } 415 \text { — \#9 }
$$

Plotting graphs is another topic which is suitable for developing the critical attitude. Before the following exercise we plotted implicitly the Descartes leaf in order to have an idea of the shape of the curve.

Exercise 8. Plot the (parametrized) Descartes leaf:

$$
x(t)=\frac{3 t}{1+t^{3}}, y(t)=\frac{3 t^{2}}{1+t^{3}} .
$$

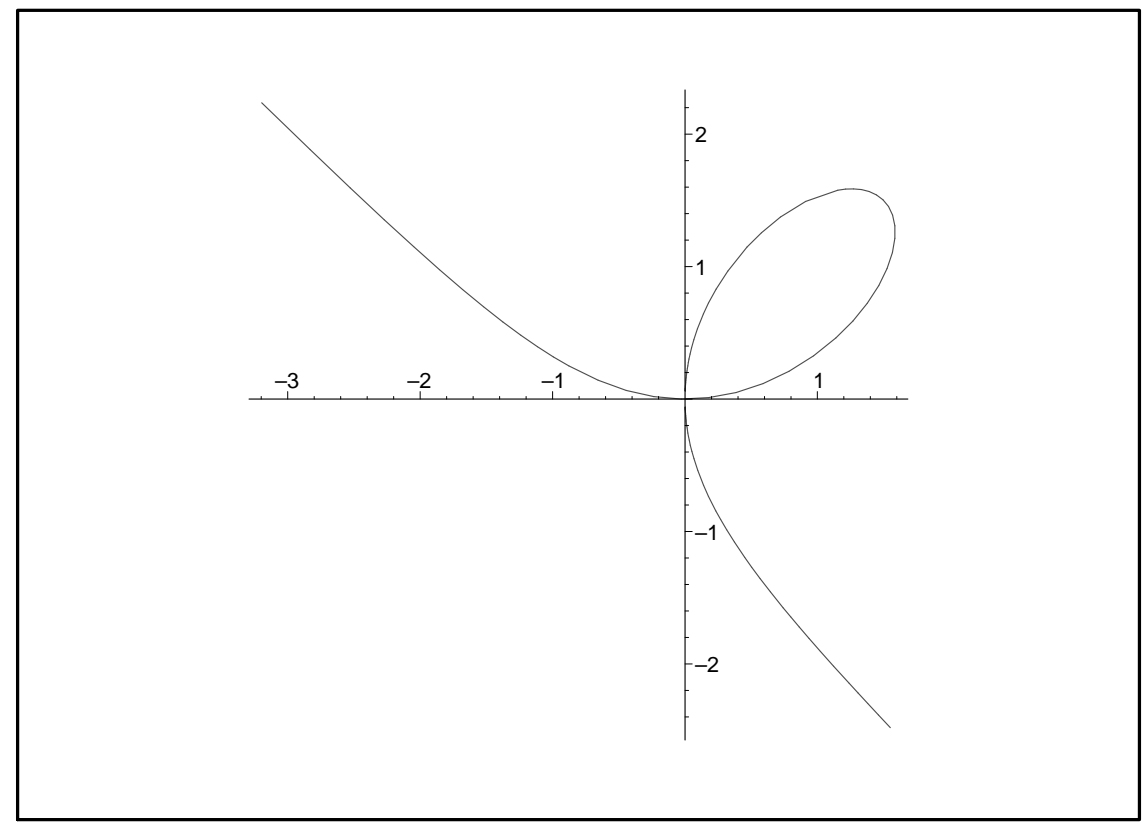

Figure 5

Without recognising the singularity at $t=-1$ one cannot get an acceptable picture. But this is not the basic point of drawing. In my experience students cannot easily find the appropriate interval for drawing the picture, in most cases my explicit hint is necessary: Which part of the curve is controlled by parameters 'near' $t=-1$ and 'near' $\pm \infty$ ? The limits

$$
\begin{aligned}
\lim _{x \rightarrow-1-0} \frac{3 t}{1+t^{3}} & =\infty, \quad \lim _{x \rightarrow-1-0} \frac{3 t^{2}}{1+t^{3}}=-\infty, \\
\lim _{x \rightarrow \infty} \frac{3 t}{1+t^{3}} & =0, \quad \lim _{x \rightarrow \infty} \frac{3 t^{2}}{1+t^{3}}=0 \quad \text { etc. }
\end{aligned}
$$


show that relatively large interval around -1 can be omitted but the graphic around the origin is controlled by reals with 'large' absolute values.

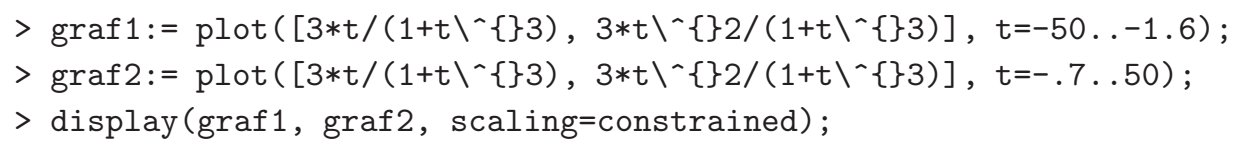

(See Figure 5.)

Give your students such type of exercises which they should know at first glance without CAS, and what is more, to force the appropriate answer from CAS, an explicit hint of the user also becomes necessary.

EXERCISE 9. Compute the 8-th power of the following matrices:

$$
A(\alpha)=\left(\begin{array}{cc}
\cos (\alpha) & -\sin (\alpha) \\
\sin (\alpha) & \cos (\alpha)
\end{array}\right) \quad B(\alpha)=\left(\begin{array}{cc}
\cos (\alpha) & \sin (\alpha) \\
\sin (\alpha) & -\cos (\alpha)
\end{array}\right) .
$$

As the student recognizes the rotation and the reflection matrices, she/he promptly says the answer:

$$
(A(\alpha))^{n}=A(n \alpha), \quad(B(\alpha))^{2 k}=E, \quad(B(\alpha))^{2 k+1}=B(\alpha), \quad n, k \in \mathbb{N} .
$$

Note that Maple evaluates the matrix multiplication directly and, even after simplification, gives a labyrinthine form. (One should simplify elements of the matrix separately.)

\section{Summary}

My intention was to give the reader some ideas on using CAS wisely in an introductory course. Clearly, there is a lot more points in this topic than just what I presented here. My operational philosophy can be articulated as follows.

- You must doubt. Check your result.

- Before pushing the Enter, have an idea concerning the result. Compare always the output with your expectation.

- Explain the result: compare with your knowledge, do a simple case by hand.

- Change the parameters (if it is possible), CAS needs your help: you work together with CAS.

- Sometimes the life is easier without CAS. Do not use it when it is not necessary. 


$$
\text { "kovacs" — 2008/4/9 — 10:31 — page } 417 \text { — \#11 }
$$

- Do not neglect paper and pencil skills.

At a more advanced level many other aspects appear see e.g. [2, 3]. An interesting case-study of analogous flavour is in [6].

\section{References}

[1] R. M. Corless, Essential Maple 7. An Introduction for Scientific Programmers, Springer, 2002.

[2] Z. Kovács and L. Kozma, Assimilation of mathematical knowledge using Maple, Teaching Mathematics and Computer Science (2003), 321-331.

[3] R. Lopez, Tips for Maple instructors, MapleTech 3, no. 2 (1996), 54-60.

[4] L. Szilassi, The right for doubting - and the necessity of doubt, in: Computer Algebra Systems and Dynamic Geometry Systems in Mathematics Teaching, (C. Sárvári, ed.), University of Pécs, 2004, 78-96.

[5] E. Talvila, Some divergent trigonometric integrals, Amer. Math. Monthly 108, no. 5 (May 2001), 432-436.

[6] W. Wade, A dialogue between calculator and algebra, Math. Teacher (NCTM) 99 (2006), 391-393.

ZOLTÁN KOVÁCS

INSTITUTE OF MATHEMATICS AND COMPUTER SCIENCE

COLLEGE OF NYÍREGYHÁZA

4401 NYIREGYHAZA

PF. 166.

HUNGARY

E-mail: kovacsz@nyf.hu

(Received October, 2007) 